\title{
A SUBGAUSSIAN EMBEDDING THEOREM
}

\author{
BY \\ SHAHAR MENDELSON* \\ Centre for Mathematics and its Applications, The Australian National University, \\ Canberra, ACT 0200, Australia \\ and Department of Mathematics, Technion, \\ I.I.T. Haifa 32000, Israel \\ e-mail: shahar.mendelson@anu.edu.au \\ AND \\ Nicole TOMCZAK-JAEGERMAnN** \\ Department of Mathematical and Statistical Sciences, University of Alberta, \\ Edmonton, Alberta, Canada T6G 2 G1 \\ e-mail:nicole@ellpspace.math.ualberta.ca
}

ABSTRACT

We prove a subgaussian extension of a Gaussian result on embedding subsets of a Euclidean space into normed spaces. Using the concentration of a random subgaussian vector around its mean we obtain an isomorphic (rather than almost isometric) result, under an additional cotype assumption on the normed space considered.

\section{Introduction}

The motivation behind this paper is the following Gaussian result on embedding subsets of a Euclidean space into normed spaces. Let $\left(g_{i}\right)$ and $\left(g_{i j}\right)$ be independent standard Gaussian random variables and set $\left(e_{i}\right)_{i=1}^{k}$ to be the standard

\footnotetext{
* Partially supported by an Australian Research Council Discovery grant.

** This author holds the Canada Research Chair in Geometric Analysis. Received June 17, 2006 and in revised form October 09, 2006
} 
unit vector basis in $\mathbb{R}^{k}$. We denote by $|\cdot|$ the Euclidean norm on $\mathbb{R}^{k}$ and by $S^{n-1}$ the Euclidean sphere in $\mathbb{R}^{n}$.

Theorem 1.1: There exists an absolute constant $c$ for which the following holds. Set $n, k \geq 1$, consider $T \subset S^{n-1}$ and let $E=\left(\mathbb{R}^{k},\|\|_{E}\right)$ be a normed space such that for every $x \in \mathbb{R}^{k},\|x\|_{E} \leq|x|$. Fix $\varepsilon>0$ and assume that

$$
\mathbb{E} \sup _{t \in T}\left|\sum_{i=1}^{n} g_{i} t_{i}\right| \leq c \varepsilon \mathbb{E}\left\|\sum_{i=1}^{k} g_{i} e_{i}\right\|_{E} .
$$

If the random operator $\Gamma: \mathbb{R}^{n} \rightarrow \mathbb{R}^{k}$ is defined by $\Gamma t=\sum_{i=1}^{k} \sum_{j=1}^{n} g_{i j} t_{j} e_{i}$, then there is a realization of $\Gamma$ such that for every $t \in T$,

$$
(1-\varepsilon) \mathbb{E}\left\|\sum_{i=1}^{k} g_{i} e_{i}\right\|_{E} \leq\left\|\left.\Gamma t\right|_{E} \leq(1+\varepsilon) \mathbb{E}\right\| \sum_{i=1}^{k} g_{i} e_{i} \|_{E} .
$$

Theorem 1.1 is a simple application of the Gaussian min-max theorem proved originally by Y. Gordon in [G1] and is an easy modification of the method of the proof of Dvoretzky's theorem and other applications in [G1] and [G2] (see also [G3]). The theorem has been recently rediscovered by G. Schechtman [S] who has given it a new proof using the majorizing measures approach. Another proof is an unpublished argument by G. Pisier [Pi4], based on his Gaussian concentration measure theorem (cf. e.g., [Pi3]). The formulation of Theorem 1.1 is taken from $[\mathrm{S}]$. One should note that all three approaches are limited to the case where $\Gamma$ is a Gaussian operator.

In this article we show that one can use the concentration of a random vector $\|\Gamma t\|_{E}$ around its mean to prove an isomorphic (rather than almost isometric) analog of Theorem 1.1, where the Gaussian operator is replaced by an arbitrary subgaussian operator, under a cotype assumption on the space $E$.

To explain the notion of a subgaussian operator we use here, recall that the $\psi_{2}$ norm of a random variable $X$ is defined by

$$
\|X\|_{\psi_{2}}=\inf \left\{u>0: \mathbb{E} \exp \left(|X|^{2} / u^{2}\right) \leq 2\right\} .
$$

Let $\mu$ be a symmetric measure on $\mathbb{R}^{n}$ which satisfies that for every $t \in \mathbb{R}^{n}$, $\mathbb{E}\langle X, t\rangle^{2}=|t|^{2}$ and $\|\langle X, t\rangle\|_{\psi_{2}} \leq L|t|$. In other words, $\mu$ is a symmetric isotropic measure on $\mathbb{R}^{n}$ and linear functionals exhibit a subgaussian decay of the order of $\exp \left(-c u^{2} / L^{2}\right)$ where $c$ is an absolute constant and $u \geq 1$. 
The operator $\Gamma: \mathbb{R}^{n} \rightarrow \mathbb{R}^{k}$ is said to be subgaussian with a constant $L$ if $\Gamma=\sum_{i=1}^{k}\left\langle X_{i}, \cdot\right\rangle e_{i}$, where $\left(X_{i}\right)_{i=1}^{k}$ are independent random vectors, distributed according to a $L$ subgaussian measure on $\mathbb{R}^{n}$.

Let us recall the concept of cotype of a Banach space which plays a key role in our approach. A Banach space $E$ has cotype $q \geq 2$ with a constant $\beta_{q}$ if for all finite sequences $\left(z_{i}\right)$ in $E$,

$$
\left(\sum_{i}\left\|z_{i}\right\|_{E}^{q}\right)^{1 / q} \leq \beta_{q} \mathbb{E}\left\|\sum_{i} \varepsilon_{i} z_{i}\right\|_{E}
$$

where $\left(\varepsilon_{i}\right)_{i}$ is a sequence of independent Bernoulli random variables. Now let us formulate our main result.

Theorem 1.2: There exist absolute constants $c_{1}, c_{2}, c_{3}>0$ for which the following holds. Set $n, k \geq 1$, consider $T \subset S^{n-1}$ and let $E=\left(\mathbb{R}^{k},\|\|_{E}\right)$ be a normed space satisfying $\|x\|_{E} \leq|x|$, for $x \in \mathbb{R}^{k}$. Fix $L>0$, assume that $E$ has cotype $q$ with a constant $\beta_{q}$ and that

$$
\mathbb{E} \sup _{t \in T}\left|\sum_{i=1}^{n} g_{i} t_{i}\right| \leq c\left(L, q, \beta_{q}\right) \mathbb{E}\left\|\sum_{i=1}^{k} g_{i} e_{i}\right\|_{E} / \sqrt{\log k},
$$

where $c\left(L, q, \beta_{q}\right):=c_{3} / L^{2} \beta_{q}^{2} \sqrt{q}$.

If $\Gamma$ is a subgaussian operator with a constant $L$ then, with probability close to 1 , for every $t \in T$

$$
c^{\prime}\left(L, q, \beta_{q}\right) \mathbb{E}\left\|\sum_{i=1}^{k} g_{i} e_{i}\right\|_{E} \leq\|\Gamma t\|_{E} \leq c_{2} L \mathbb{E}\left\|\sum_{i=1}^{k} g_{i} e_{i}\right\|_{E},
$$

where $c^{\prime}\left(L, q, \beta_{q}\right):=c_{1} / L \beta_{q} \sqrt{q}$. Moreover, if $\Gamma$ is an operator with independent Bernoulli random entries then the assertion of the theorem remains true with (1.2) replaced by

$$
\mathbb{E} \sup _{t \in T}\left|\sum_{i=1}^{n} g_{i} t_{i}\right| \leq c\left(L, q, \beta_{q}\right) \mathbb{E}\left\|\sum_{i=1}^{k} g_{i} e_{i}\right\|_{E} .
$$

To illustrate the main theorem we shall formulate just one result for random \pm 1 embeddings of the Euclidean space. 
Corollary 1.3: Let $E=\left(\mathbb{R}^{k},\|\|_{E}\right)$ be a normed space of cotype $q$ with a constant $\beta_{q}$ such that for every $x \in \mathbb{R}^{k},\|x\|_{E} \leq|x|$. Assume that

$$
n \leq\left(c_{1} / \beta_{q}^{2} q\right)\left(\mathbb{E}\left\|\sum_{i=1}^{k} g_{i} e_{i}\right\|_{E}\right)^{2},
$$

where $c_{1}>0$ is an absolute constant. Let $\Gamma: \mathbb{R}^{n} \rightarrow \mathbb{R}^{k}$ be a random operator with independent Bernoulli entries. Then, with probability close to 1 , the random subspace $F:=\Gamma\left(\mathbb{R}^{n}\right) \subset \mathbb{R}^{k}$ spanned by \pm 1 vectors satisfies $c^{\prime} A|z| \leq\|z\|_{E} \leq c^{\prime \prime} A|z|$ for all $z \in F$, where $A=\mathbb{E}\left\|\sum g_{i} e_{i}\right\|_{E} / \sqrt{k}$, and $c^{\prime}, c^{\prime \prime}>0$ depend on $L, q, \beta_{q}$.

In particular, if the Euclidean unit ball on $\mathbb{R}^{k}$ is the maximal volume ellipsoid for the unit ball $B_{E}$ of $E$ then the assertion holds once $n \leq\left(c_{1} / \beta_{q}^{2} q\right) k^{2 / q}$.

We end this introduction with some notation and definitions we will use throughout this note. For a finite set $A$, denote by $|A|$ its cardinality. With a minor abuse of notation, we also denote by $|\cdot|$ the fixed Euclidean structure we consider, and let $\left(e_{i}\right)$ be the standard unit vector basis with respect to that structure. If $A \subset \mathbb{R}^{m}$, let $\ell_{*}(A)=\mathbb{E} \sup _{a \in A}\left|\sum_{i=1}^{m} g_{i} a_{i}\right|$, where $\left(g_{i}\right)_{i=1}^{m}$ are independent standard Gaussian variables. Note that if $A=B_{E^{*}}$, the unit ball of the dual space to $E$, then $\ell_{*}(A)=\mathbb{E}\left\|\sum_{i=1}^{k} g_{i} e_{i}\right\|_{E}$. Finally, all absolute constants are denoted by $c, c_{1}, \ldots$. Their values may change from line to line.

Acknowledgement: The authors wish to thank Olivier Guédon, Gilles Pisier and Gideon Schechtman for several interesting conversations. They are also indebted to Gilles Pisier for pointing out a simpler proof to Lemma 2.6 than the original one. This work has begun when the second-named author visited the Australian National University, and has been concluded when the both authors participated in the Trimester "Phenomena in High Dimensions" at the Centre Emile Borel in the Institute Henri Poincaré. They are grateful to these institutions for their hospitality and an excellent working atmosphere.

\section{Preliminaries - Subgaussian vectors}

We will be interested in independent, symmetric random variables that exhibit a subgaussian tail behaviour. We begin this section by recalling some of the basic facts concerning random variables with certain decay properties. For basic properties of such random variables we refer the reader to [LTa, DG]. 
Definition 2.1: For $\alpha \geq 1$ the $\psi_{\alpha}$ norm, of a random variable $X$ is defined by

$$
\|X\|_{\psi_{\alpha}}=\inf \left\{\lambda>0: \mathbb{E} \exp \left(|X|^{\alpha} / \lambda^{\alpha}\right) \leq 2\right\} .
$$

It is standard to verify that if $X$ has a bounded $\psi_{\alpha}$ norm, then for $t \geq 1$

$$
\mathbb{P}(|X| \geq t) \leq 2 \exp \left(-c t^{\alpha} /\|X\|_{\psi_{\alpha}}^{\alpha}\right)
$$

where $c$ is an absolute constant. The converse is also true, and if $X$ has a tail bounded by $\exp \left(-t^{\alpha} / K^{\alpha}\right)$ then $\|X\|_{\psi_{\alpha}} \leq c_{1} K$.

Another basic property of the $\psi_{2}$ norm is that if $X_{1}, \ldots, X_{n}$ are independent and centered then

$$
\left\|\sum_{i=1}^{n} a_{i} X_{i}\right\|_{\psi_{2}} \leq c\left(\sum_{i=1}^{n} a_{i}^{2}\left\|X_{i}\right\|_{\psi_{2}}^{2}\right)^{1 / 2}
$$

where $c$ is an absolute constant. If $\left(X_{i}\right)_{i=1}^{n}$ are symmetric, (2.1) follows from an easy calculation using the moment generating function of the sum and the fact that for every $t>0, \mathbb{E} \exp (t X) \leq \exp \left(t^{2} b^{2}\right)$, where $b=c_{1}\|X\|_{\psi_{2}}$. The extension of (2.1) from the symmetric case to the centered one is evident from a standard symmetrization argument.

We require two additional preliminary results. First, a bound due to Pisier [Pi1] (cf. also [VW] Section 2.2) on the $\psi_{\alpha}$ norm of a maximum of $k$ random variables, which we formulate only for $\alpha=1$.

Lemma 2.2: There exists an absolute constant $c$ for which the following holds. Let $X_{1}, \ldots, X_{k}$ be random variables. Then,

$$
\left\|\max _{1 \leq i \leq k}\left|X_{i}\right|\right\|_{\psi_{1}} \leq c \max _{1 \leq i \leq k}\left\|X_{i}\right\|_{\psi_{1}} \log k .
$$

The second preliminary result deals with the $\psi_{1}$ behaviour of a sum of independent $\psi_{1}$ random variables, and is due to Talagrand (see, e.g. [LTa], Theorem $6.21)$.

Theorem 2.3: There exists an absolute constant $c$ such that if $X_{1}, \ldots, X_{k}$ are independent, centered random variables then

$$
\left\|\sum_{i=1}^{k} X_{i}\right\|_{\psi_{1}} \leq c\left(\left\|\sum_{i=1}^{k} X_{i}\right\|_{L_{1}}+\left\|\max _{1 \leq i \leq k}\left|X_{i}\right|\right\|_{\psi_{1}}\right) .
$$

Consider a random process $\left\{Z_{t}: t \in T\right\}$ indexed by a metric space $(T, d)$. We say that the process is subgaussian with respect to the metric $d$ if it satisfies 
the increment condition that for every $x, y \in T$ and every $u \geq 1$,

$$
\mathbb{P}\left(\left|Z_{x}-Z_{y}\right| \geq u d(x, y)\right) \leq 2 \exp \left(-u^{2} / 2\right)
$$

(the constants 2 and $1 / 2$ can be replaced by any other fixed constants, as can be the restriction $u \geq 1$ ).

The generic chaining mechanism allows one to obtain an exponential deviation inequality for $\sup _{t \in T}\left|Z_{t}-Z_{t_{0}}\right|$ where $t_{0}$ is an arbitrary point in $T$ using an increment condition. To that end we require the definition of the $\gamma_{2}$ functional [Ta3].

Definition 2.4: Let $(T, d)$ be a metric space. An admissible sequence of $T$ is a collection of subsets of $T,\left\{T_{s}: s \geq 0\right\}$, such that for every $s \geq 1,\left|T_{s}\right|=2^{2^{s}}$ and $\left|T_{0}\right|=1$. Define the $\gamma_{2}$ functional by

$$
\gamma_{2}(T, d)=\inf \sup _{t \in T} \sum_{s=0}^{\infty} 2^{s / 2} d\left(t, T_{s}\right),
$$

where $d\left(t, T_{s}\right)$ is the distance between the set $T_{s}$ and $t$, and the infimum is taken with respect to all admissible sequences of $T$.

It is well-known that the $\gamma_{2}(T)$ functional, with respect to a Euclidean metric, is connected to the behaviour of a Gaussian process indexed by $T$. Indeed, let $\left\{G_{t}: t \in T\right\}$ be a centered Gaussian process indexed by a set $T$, and for every $s, t \in T$, let $d^{2}(s, t)=\mathbb{E}\left|G_{s}-G_{t}\right|^{2}$. Then

$$
c_{1} \gamma_{2}(T, d) \leq \mathbb{E} \sup _{t \in T}\left|G_{t}\right| \leq c_{2} \gamma_{2}(T, d),
$$

where $c_{1}$ and $c_{2}$ are absolute constants. The upper bound is due to Fernique $[\mathrm{F}]$ and the lower bound is Talagrand's majorizing measure theorem [Ta1]. The proof of both parts and the most recent survey on the topic can be found in [Ta3].

Let us state a typical deviation estimate that follows from a generic chaining argument. Proofs of a similar flavor can also be found in [Ta3, Chapter 1].

Theorem 2.5: There exists an absolute constant $c$ for which the following holds. Let $(T, d)$ be a metric space and assume the process $\left\{Z_{t}: t \in T\right\}$ is subgaussian with respect to the metric $d$. Then, for any $u \geq 4$ and any $t_{0} \in T$,

$$
\mathbb{P}\left(\sup _{t \in T}\left|Z_{t}-Z_{t_{0}}\right| \geq u \gamma_{2}(T, d)\right) \leq 2 \exp \left(-c u^{2}\right) .
$$


Let $\left(\xi_{i}\right)_{i=1}^{k}$ be i.i.d. symmetric random variables which satisfy the subgaussian condition $\|\xi\|_{\psi_{2}} \leq L$. We are interested in the moments of the random variable $\left\|\sum_{i=1}^{k} \xi_{i} e_{i}\right\|_{E}$, where \|\|$_{E}$ is a norm on $\mathbb{R}^{k}$. An important part in our argument is the fact that under a cotype assumption on $E$, these moments are equivalent to the analogous Gaussian moments.

The first observation in this direction compares the $L_{p}(E)$ norm of a subgaussian vector with the $L_{1}(E)$ norm of a Gaussian one. The proof we present here is due to Jain and Marcus [JM]. It was shown to us by G. Pisier and we present it for the sake of completeness.

LEMma 2.6: There exists an absolute constant $c$ for which the following holds. Let $p \geq 1$ and set $\left(\xi_{i}\right)_{i=1}^{k}$ to be independent symmetric random variables which satisfy that $\mathbb{E} \xi_{i}^{2}=1$ and $\left\|\xi_{i}\right\|_{\psi_{2}} \leq L$. Then

$$
\left\|\sum_{i=1}^{k} \xi_{i} e_{i}\right\|_{L_{p}(E)} \leq c L \sqrt{p}\left\|\sum_{i=1}^{k} g_{i} e_{i}\right\|_{L_{1}(E)} .
$$

Proof. Let $\xi$ be a symmetric $L$ subgaussian random variable. Then there is a centered Gaussian variable denoted by $Y$ with variance $\sigma_{Y}^{2}=c L^{2}$, such that for every $t \geq 1$,

$$
\mathbb{P}(|\xi| \geq t) \leq \mathbb{P}(|Y| \geq t)
$$

Let $\xi^{*}$ and $Y^{*}$ be the decreasing rearrangements of $\xi$ and $Y$, respectively, defined on $[0,1]$. That is, $\xi^{*}(x)=\inf \{t: \mathbb{P}(|\xi|>t) \leq x\}$ for all $x \in[0,1]$, and $Y^{*}$ is defined similarly. Note that $Y^{*}$ is continuous on $[0,1]$ and for every $x \in[0,1]$, $\xi^{*}(x) \leq 1+Y^{*}(x)$.

Indeed, observe that if $f^{*}$ is a decreasing rearrangement of $f$ then for every $t, f^{*}(\mathbb{P}(|f| \geq t)) \leq t$, and the equality holds if $f^{*}$ is continuous at the point $x=\mathbb{P}(|f| \geq t)$.

Now, for $t \geq 1$ set $x_{t}:=\mathbb{P}(|\xi| \geq t)$ and $y_{t}:=\mathbb{P}(|Y| \geq t)$. Then $y_{t} \geq x_{t}$ and $\xi^{*}\left(x_{t}\right) \leq Y^{*}\left(y_{t}\right) \leq Y^{*}\left(x_{t}\right)$. This implies that $\xi^{*}(x) \leq Y^{*}(x)$ for all $x \in\left[0, x_{1}\right]$, where $x_{1}=\mathbb{P}(|\xi| \geq 1)$. For $x \geq x_{1}, \xi^{*}(x) \leq 1$, and $Y^{*}$ is non-negative. The combination of the two observations gives the desired inequality.

Let $\left(\xi_{i}\right)_{i=1}^{k}$ be as in the lemma and consider $\left(\xi_{i}^{*}\right)_{i=1}^{k}$. If $\left(\varepsilon_{i}\right)_{i=1}^{k}$ are independent Bernoulli variables then $\varepsilon_{i} \xi_{i}^{*}$ has the same distribution as $\xi_{i}$, for $i=1, \ldots, k$. A similar fact holds for $\left(Y_{i}^{*}\right)_{i=1}^{k}$ and $\left(Y_{i}\right)_{i=1}^{k}$. 
By the Kahane-Khintchine inequality and the contraction principle for Bernoulli processes (see, e.g. [LTa]), for every $p \geq 1$,

$\mathbb{E}\left\|\sum_{i=1}^{k} \xi_{i} e_{i}\right\|_{E}^{p}=\int_{0}^{1} \mathbb{E}_{\varepsilon}\left\|\sum_{i=1}^{k} \varepsilon_{i} \xi_{i}^{*}(x) e_{i}\right\|_{E}^{p} \leq c p^{p / 2} \int_{0}^{1} \mathbb{E}_{\varepsilon}\left\|\sum_{i=1}^{k} \varepsilon_{i}\left(Y_{i}^{*}(x)+1\right) e_{i}\right\|_{E}$.

Hence,

$$
\left\|\sum_{i=1}^{k} \xi_{i} e_{i}\right\|_{L_{p}(E)} \leq c \sqrt{p}\left(\left\|\sum_{i=1}^{k} \varepsilon_{i} e_{i}\right\|_{L_{1}(E)}+\left\|\sum_{i=1}^{k} Y_{i} e_{i}\right\|_{L_{1}(E)}\right) .
$$

The claim now follows from the definition of $Y$ and the fact that Rademacher averages are dominated by Gaussian ones (see, e.g. [LTa] or [MS], Appendix II).

Lemma 2.6 provides the "general" direction in the equivalence between the $L_{p}(E)$ norms of subgaussian and Gaussian vectors - that holds without any structural assumptions on $E$. The reverse direction is not true for an arbitrary space $E$, but rather, requires cotype assumptions. The following theorem was proved in [MaPi] (see also [Pi2]). The proof for the Rademacher case can be found in Appendix II of [MS]. It is straightforward to check that only minimal modifications to that proof are needed to show that it actually holds for an arbitrary subgaussian symmetric variable, and thus we omit the proof.

Theorem 2.7: For every $p \geq 1$ there exists a constant $c(p)$ for which the following holds. Let $E$ be a Banach space of cotype $q$ with a constant $\beta_{q}$. Let $\left(\xi_{i}\right)_{i=1}^{k}$ be independent symmetric random variables such that $\mathbb{E} \xi_{i}^{2}=1$ and $\left\|\xi_{i}\right\|_{\psi_{2}} \leq L$. Then, for every $x_{1}, \ldots, x_{k} \in E$,

$$
\left\|\sum_{i=1}^{k} g_{i} x_{i}\right\|_{L_{p}(E)} \leq c(p) L \sqrt{q} \beta_{q}\left\|\sum_{i=1}^{k} \xi_{i} x_{i}\right\|_{L_{p}(E)} .
$$

Moreover, $c(p)$ is an absolute constant for $p \leq q$.

For our needs it suffices to obtain an equivalence result for $p=1$.

Corollary 2.8: There exist absolute constants $c_{1}$ and $c_{2}$ for which the following holds. Consider $\left(\xi_{i}\right)_{i=1}^{k}$ as above and assume that $E$ has cotype $q$ with a constant $\beta_{q}$. Then,

$$
\left(c_{1} / L\right)\left\|\sum_{i=1}^{k} \xi_{i} e_{i}\right\|_{L_{1}(E)} \leq\left\|\sum_{i=1}^{k} g_{i} e_{i}\right\|_{L_{1}(E)} \leq c_{2} L \sqrt{q} \beta_{q}\left\|\sum_{i=1}^{k} \xi_{i} e_{i}\right\|_{L_{1}(E)} .
$$




\section{The main result}

The proof of Theorem 1.2 is based on three ingredients. The first one is a concentration result for each one of the random variables

$$
Z_{t}=\left\|\sum_{i=1}^{k}\left\langle X_{i}, t\right\rangle e_{i}\right\|_{E}
$$

around its mean.

Definition 3.1: Let $k \geq 1$ and set $\left(\xi_{i}\right)_{i=1}^{k}$ to be independent random variables with distribution $\xi$. A space $E=\left(\mathbb{R}^{k},\|\|_{E}\right)$ is $\alpha$ concentrated with respect to $\xi$ if

$$
\begin{aligned}
\mathbb{P}\left(\left\|\left|\sum_{i=1}^{k} \xi_{i} e_{i}\left\|_{E}-\mathbb{E}\right\| \sum_{i=1}^{k} \xi_{i} e_{i}\left\|_{E} \mid \geq \frac{1}{2} \mathbb{E}\right\|\right.\right.\right. & \left.\sum_{i=1}^{k} \xi_{i} e_{i} \|_{E}\right) \\
& \leq 2 \exp \left(-\frac{\left(\mathbb{E}\left\|\sum_{i=1}^{k} \xi_{i} e_{i}\right\|_{E}\right)^{2}}{\alpha^{2}}\right) .
\end{aligned}
$$

The concentration result for Gaussian or Rademacher vectors (see e.g. [LTa]) shows that if for every $x \in E,\|x\|_{E} \leq|x|$, then $E$ satisfies Definition 3.1 for $\alpha$ which is an absolute constant. We will show later that a slightly weaker estimate $\alpha \sim L \sqrt{\log k}$ holds for an arbitrary $k$-dimensional space and any $L$ subgaussian random variable. The concentration assumption, combined with an approximation argument, leads to the following technical embedding result.

Theorem 3.2: There exist absolute constants $c_{1}$ and $c_{2}$ for which the following holds. Let $\mu$ be an isotropic $\psi_{2}$ probability measure on $\mathbb{R}^{n}$ with a constant $L$ and set $X$ to be distributed according to $\mu$. Let $\Gamma$ be the corresponding operator, i.e., $\Gamma t=\sum_{i=1}^{k}\left\langle X_{i}, t\right\rangle e_{i}$ for $t \in \mathbb{R}^{n}$. Consider $T \subset S^{n-1}$ and for every $t \in T$ denote $\xi_{t}=\langle X, t\rangle$.

Let $E$ be a $k$ dimensional space which is $\alpha$ concentrated with respect to $\xi_{t}$ for every $t \in T$. Fix $\varepsilon>0$ and set

$$
H:=L\left(\ell_{*}(T)+\varepsilon \mathbb{E}\left\|\sum_{i=1}^{k} g_{i} e_{i}\right\|_{E}\right)
$$


and $p_{0}:=\exp \left(c_{1} \ell_{*}^{2}(T) / \varepsilon^{2}-\inf _{t \in T}\left(\mathbb{E}\left\|\sum_{i=1}^{k}\left\langle X_{i}, t\right\rangle e_{i}\right\|_{E}\right)^{2} / \alpha^{2}\right)$. Then, for every $s \geq 1$, with probability at least $1-p_{0}-2 \exp \left(-s^{2}\right)$, we have, for every $t \in T$,

$$
\frac{1}{2} \mathbb{E}\|\Gamma t\|_{E}-c_{2} s H \leq\|\Gamma t\|_{E} \leq \frac{3}{2} \mathbb{E}\|\Gamma t\|_{E}+c_{2} s H .
$$

Proof. Let $\Lambda$ be an $\varepsilon$-net of $T$ with respect to the Euclidean norm $|\cdot|$. By Sudakov's inequality (cf. e.g., [Pi3]), $\log |\Lambda| \leq c_{1} \ell_{*}^{2}(T) / \varepsilon^{2}$. Applying the $\alpha$ concentration assumption,

$$
\begin{aligned}
\mathbb{P}(\exists v & \left.\in \Lambda:\left|\left\|\sum_{i=1}^{k}\left\langle X_{i}, v\right\rangle e_{i}\right\|_{E}-\mathbb{E}\left\|\sum_{i=1}^{k}\left\langle X_{i}, v\right\rangle e_{i}\right\|_{E}\right| \geq \frac{1}{2} \mathbb{E}\left\|\sum_{i=1}^{k}\left\langle X_{i}, v\right\rangle e_{i}\right\|_{E}\right) \\
& \leq \sum_{v \in \Lambda} \exp \left(-\frac{\left(\mathbb{E}\left\|\sum_{i=1}^{k}\left\langle X_{i}, v\right\rangle e_{i}\right\|_{E}\right)^{2}}{\alpha^{2}}\right) \\
& \leq \exp \left(c_{1} \frac{\ell_{*}^{2}(T)}{\varepsilon^{2}}-\frac{\inf _{t \in T}\left(\mathbb{E}\left\|\sum_{i=1}^{k}\left\langle X_{i}, t\right\rangle e_{i}\right\|_{E}\right)^{2}}{\alpha^{2}}\right)=p_{0} .
\end{aligned}
$$

Set $U:=(T-T) \cap \varepsilon B_{2}^{k}$ and observe that $T \subset \Lambda+U$. Thus by an easy approximation argument the theorem will follow from the above estimate, the lemma below and the fact that $\ell_{*}(T-T) \leq 2 \ell_{*}(T)$.

The proof of the following lemma is based on a standard generic chaining argument [Ta3].

Lemma 3.3: Let $\tilde{T} \subset \mathbb{R}^{n}, \varepsilon>0, \tilde{U}:=\tilde{T} \cap \varepsilon B_{2}^{n}$ and consider $E$ and $\Gamma$ to be as in Theorem 3.2. Then, for any $s \geq 1$,

$$
\mathbb{P}\left(\sup _{u \in \tilde{U}}\|\Gamma u\|_{E} \leq c_{3} s \tilde{H}\right) \geq 1-2 \exp \left(-s^{2}\right),
$$

where $\tilde{H}$ is given by (3.1), by replacing $T$ by $\tilde{T}$, and $c_{3}>0$ is an absolute constant.

Proof. Let $B_{E^{*}}$ denote the unit ball of $E^{*}$ and consider the process

$$
(u, y) \rightarrow Z_{u, y}:=\sum_{i=1}^{k}\left\langle X_{i}, u\right\rangle\left\langle e_{i}, y\right\rangle .
$$

indexed by $\tilde{U} \times B_{E^{*}}$. Clearly, $\sup _{\left\{u \in \tilde{U}, y \in B_{\left.E^{*}\right\}}\right.} Z_{u, y}=\sup _{u \in \tilde{U}}\|\Gamma u\|_{E}$. Note that $Z$ is centered and subgaussian with respect to the appropriate metric. Indeed, 
the increment associated with the elements $(u, y)$ and $\left(u_{1}, y_{1}\right)$ satisfies

$$
\begin{aligned}
\left|Z_{u, y}-Z_{u_{1}, y_{1}}\right| & \leq\left|Z_{u, y}-Z_{u, y_{1}}\right|+\left|Z_{u, y_{1}}-Z_{u_{1}, y_{1}}\right| \\
& =\left|\sum_{i=1}^{k}\left\langle X_{i}, u\right\rangle\left\langle y-y_{1}, e_{i}\right\rangle\right|+\left|\sum_{i=1}^{k}\left\langle X_{i}, u-u_{1}\right\rangle\left\langle y_{1}, e_{i}\right\rangle\right| .
\end{aligned}
$$

Using (2.1) we can bound the $\psi_{2}$ norm of the first term by

$$
\|\langle X, u\rangle\|_{\psi_{2}}\left(\sum_{i=1}^{k}\left\langle y-y_{1}, e_{i}\right\rangle^{2}\right)^{1 / 2} \leq L|u| \cdot\left|y-y_{1}\right| \leq L \varepsilon\left|y-y_{1}\right|,
$$

where the last inequality follows from $\tilde{U} \subset \varepsilon B_{2}^{k}$.

By a similar argument, the second term has a $\psi_{2}$ norm at most $L\left|u-u_{1}\right|\left|y_{1}\right| \leq$ $L\left|u-u_{1}\right|$, because $\left\|y_{1}\right\|_{E^{*}} \leq 1$ and since the condition $\|x\|_{E} \leq|x|$ for every $x$ implies $|y| \leq\|y\|_{E^{*}}$ for every $y$. Hence, adding the two probabilities corresponding to the two terms above, it follows that for every $s \geq 1$,

$$
\mathbb{P}\left(\left|Z_{u, y}-Z_{u_{1}, y_{1}}\right| \geq c_{4} s L\left(\varepsilon\left|y-y_{1}\right|+\left|u-u_{1}\right|\right)\right) \leq 2 \exp \left(-s^{2}\right) .
$$

Applying a generic chaining argument combined with the majorizing measures Theorem,

$$
\begin{aligned}
\sup _{u, y}\left|Z_{u, y}\right| & \leq c L\left(\gamma_{2}(\tilde{U},|\cdot|)+\varepsilon \gamma_{2}\left(B_{E^{*}},|\cdot|\right)\right) \\
& \leq c_{3} L\left(\ell_{*}(\tilde{U})+\varepsilon \mathbb{E}\left\|\sum_{i=1}^{k} g_{i} e_{i}\right\|_{E}\right) \leq c_{3} \tilde{H},
\end{aligned}
$$

and moreover, for every $s \geq 1$

$$
\mathbb{P}\left(\sup _{u, y}\left|Z_{u, y}\right| \geq c_{3} s \tilde{H}\right) \leq 2 \exp \left(-s^{2}\right)
$$

from which the claim follows. This also completes the proof of Theorem 3.2 .

Remark 3.4: Let us note that in the Gaussian case, a version of Lemma 3.3 (with probability larger than or equal to $1-1 / s$ ) is a consequence of Chevet's inequality (based on Slepian's lemma) followed by Chebyshev's inequality.

In order to apply Theorem 3.2, we shall need to control probability $p_{0}$ and the expressions appearing in (3.2). This will follow from the third and the final 
fact we require, namely, that if $E$ has cotype $q$ with a constant $\beta_{q}$ and $T \subset S^{n-1}$ then

$$
\begin{aligned}
\frac{c_{5}}{L} \sup _{t \in T} \mathbb{E}\left\|\sum_{i=1}^{k}\left\langle X_{i}, t\right\rangle e_{i}\right\|_{E} & \leq \mathbb{E}\left\|\sum_{i=1}^{k} g_{i} e_{i}\right\|_{E} \\
& \leq c_{4} L \sqrt{q} \beta_{q} \inf _{t \in T} \mathbb{E}\left\|\sum_{i=1}^{k}\left\langle X_{i}, t\right\rangle e_{i}\right\|_{E},
\end{aligned}
$$

for suitable absolute constants $c_{4}, c_{5}$. This is evident by applying Corollary 2.8 for $\xi_{i}=\left\langle X_{i}, t\right\rangle$.

Thus, all that is needed for the proof of Theorem 1.2 is to show an appropriate concentration result for an arbitrary subgaussian measure.

Let us mention once again that for the Gaussian of the Rademacher measures such a concentration result is well-known, and yields the estimate of $\alpha$ being an absolute constant. This will show the "moreover" part of the theorem. For the general case, consider the following

Lemma 3.5: There exists an absolute constant $c_{6}>0$ for which the following holds. Let $\left(\xi_{i}\right)_{i=1}^{k}$ be i.i.d. symmetric subgaussian random variables with $\|\xi\|_{\psi_{2}} \leq L$. Let $E=\left(\mathbb{R}^{k},\|\|_{E}\right)$, such that for every $x \in E,\|x\|_{E} \leq|x|$. Then for every $t \geq 2$,

$$
\mathbb{P}\left(\left\|\left|\sum_{i=1}^{k} \xi_{i} e_{i}\left\|_{E}-\mathbb{E}\right\| \sum_{i=1}^{k} \xi_{i} e_{i} \|_{E}\right| \geq t\right) \leq 2 \exp \left(-c_{6} t^{2} / L^{2} \log ^{2} k\right) .\right.
$$

The idea of the proof is to truncate the variables $\xi_{i}$ at the right level, that is, to split each of the $\xi_{i}$ to $\xi^{-}=\xi 1_{\{|\xi| \leq \rho\}}$ and $\xi^{+}=\xi 1_{\{|\xi|>\rho\}}$ for some fixed $\rho$. We will show that the truncated part $\left\|\sum_{i=1}^{k} \xi_{i}^{-} e_{i}\right\|_{E}$ is concentrated around its mean using Talagrand's convex distance inequality [Ta2] (see also [L], Corollary 4.10). The second part is bounded with high probability because the variables $\xi_{i}$ decay rapidly.

Proof. Consider the truncated variable $\xi^{-}=\xi 1_{\{|\xi| \leq \rho\}}$ and $\xi^{+}=\xi 1_{\{|\xi|>\rho\}}$, where $\rho$ will be determined later. The first step is to prove a concentration result for $\left\|\sum_{i=1}^{k} \xi_{i}^{-} e_{i}\right\|_{E}$ around its mean. Indeed, as a function on $[-\rho, \rho]^{k}$, $\left\|\sum_{i=1}^{k} a_{i} e_{i}\right\|_{E}$ is convex. Moreover, since $\|x\|_{E} \leq|x|$, it is 1-Lipschitz on $\left([-\rho, \rho]^{k},|\cdot|\right)$. Thus, by the convex distance inequality it follows that for every 
$t>0$

$$
\mathbb{P}\left(\left\|\left|\sum_{i=1}^{k} \xi_{i}^{-} e_{i}\left\|_{E}-\mathbb{E}\right\| \sum_{i=1}^{k} \xi_{i}^{-} e_{i} \|_{E}\right| \geq t\right) \leq \exp \left(-c_{1}^{\prime} t^{2} / \rho^{2}\right)\right.
$$

for a suitable absolute constant $c_{1}^{\prime}$.

To take care of the "unbounded" part we shall require some properties of Orlicz norms. First, by Theorem 2.3, if $X_{1}, \ldots, X_{k}$ are independent, centered random variables then

$$
\left\|\sum_{i=1}^{k} X_{i}\right\|_{\psi_{1}} \leq c_{2}^{\prime}\left(\left\|\sum_{i=1}^{k} X_{i}\right\|_{L_{1}}+|\max | X_{i} \mid \|_{\psi_{1}}\right) .
$$

Also, for any random variable $X,\left\|X^{2}\right\|_{\psi_{1}}=\|X\|_{\psi_{2}}^{2}$, and, by Lemma 2.2, if $\left(X_{i}\right)_{i=1}^{k}$ are identically distributed random variables then $\left\|\max _{1 \leq i \leq k}\left|X_{i}\right|\right\|_{\psi_{1}} \leq$ $\|X\|_{\psi_{1}} \log k$.

Let $X_{i}=\left(\xi_{i}^{+}\right)^{2}-\mathbb{E}\left(\xi_{i}^{+}\right)^{2}$. By the remarks above it is clear that $\left\|X_{i}\right\|_{\psi_{1}} \leq c_{3}^{\prime} L^{2}$ and a standard calculation yields that

$$
\left\|\sum_{i=1}^{k} X_{i}\right\|_{L_{1}} \leq c_{3}^{\prime} k\left(\rho^{2}+2 L^{2}\right) \exp \left(-c_{4}^{\prime} \rho^{2} / L^{2}\right)
$$

for suitable absolute constants $c_{3}^{\prime}$ and $c_{4}^{\prime}$. Hence, by (3.5),

$$
\left\|\sum_{i=1}^{k}\left(\xi_{i}^{+}\right)^{2}\right\|_{\psi_{1}} \leq c_{5}^{\prime}\left(k\left(\rho^{2}+2 L^{2}\right) \exp \left(-c_{4}^{\prime} \rho^{2} / L^{2}\right)+L^{2} \log k\right)
$$

for an absolute constant $c_{5}^{\prime}$.

Since $\|x\|_{E} \leq|x|$ then $\left\|\sum_{i=1}^{k} \xi_{i}^{+} e_{i}\right\|_{E} \leq\left(\sum_{i=1}^{k}\left(\xi_{i}^{+}\right)^{2}\right)^{1 / 2}$. Thus, setting $\rho=c_{6}^{\prime} L \sqrt{\log k}$,

$$
\left\|\sum_{i=1}^{k} \xi_{i}^{+} e_{i}\right\|_{\psi_{2}(E)} \leq c_{7}^{\prime} L \sqrt{\log k}, \quad \mathbb{E}\left\|\sum_{i=1}^{k} \xi_{i}^{+} e_{i}\right\|_{E} \leq 1 .
$$

Therefore, if $t \geq 2$,

$$
\mathbb{P}\left(\left\|\sum_{i=1}^{k} \xi_{i}^{+} e_{i}\right\|_{E}+\mathbb{E}\left\|\sum_{i=1}^{k} \xi_{i}^{+} e_{i}\right\|_{E} \geq t\right) \leq 2 \exp \left(-c_{8}^{\prime} t^{2} / L^{2} \log k\right) .
$$

The claim follows by combining (3.4) and (3.6). 
Proof of Theorem 1.2. Let $c_{1}, c_{2}, c_{3}, c_{4}, c_{5}, c_{6}$ denote the constants appearing earlier throughout this section. Let $\alpha$ be the constant from Definition 3.1 (we may clearly assume that $\alpha \geq 1$ ), and note that by Lemma 3.5, $\alpha \leq L \sqrt{\log k} / c_{6}$. However, for clarity, we shall make our calculations for a general $\alpha$. Let $s \geq 1$. We shall show that for every $s \geq 1$, (1.3) holds with probability $1-\tilde{p}_{0}-2 \exp \left(-s^{2}\right)$, where $\tilde{p}_{0}=\exp \left(-\tilde{c}_{4}\left(\mathbb{E}\left\|\sum_{i=1}^{k} g_{i} e_{i}\right\|_{E}\right)^{2} / \alpha^{2}\right)$ and $\tilde{c}_{4}>0$ is an absolute constant.

To this end we shall use Theorem 3.2. Fix $\varepsilon>0$ to be determined later and assume that $T$ and $E$ satisfy

$$
\ell_{*}(T) \leq\left(\varepsilon c^{\prime} / L \sqrt{q} \beta_{q} \alpha\right) \mathbb{E}\left\|\sum_{i=1}^{k} g_{i} e_{i}\right\|_{E}
$$

for some constant $0<c^{\prime}<1$. A straightforward calculation shows that if $c^{\prime}$ is sufficiently small (depending on $c_{1}, c_{4}$ ) then $p_{0}$ defined in the Theorem satisfies $p_{0} \leq \tilde{p}_{0}$, with constant $\tilde{c}_{4}$ depending only on $c_{1}, c_{4}$ and $c^{\prime}$. Furthermore,

$$
H \leq\left(\varepsilon / \sqrt{q} \beta_{q}\right)\left(\left(c^{\prime} / \alpha\right)+L \sqrt{q} \beta_{q}\right) \mathbb{E}\left\|\sum_{i=1}^{k} g_{i} e_{i}\right\|_{E} .
$$

Combining this with (3.2) and (3.3) it follows that

$$
\|\Gamma t\|_{E} \geq\left(\frac{1}{2 c_{4} L \sqrt{q} \beta_{q}}-\frac{c_{2} s \varepsilon}{\sqrt{q} \beta_{q}}\left(\frac{c^{\prime}}{\alpha}+L \sqrt{q} \beta_{q}\right)\right) \mathbb{E}\left\|\sum_{i=1}^{k} g_{i} e_{i}\right\|_{E} .
$$

Letting $\varepsilon=c^{\prime \prime} /\left(s L^{2} \sqrt{q} \beta_{q}\right)$ for an appropriate absolute constant $c^{\prime \prime}>0$, and noting that $L, q$ and $\beta_{q}$ are greater than 1 , we conclude the left hand side of (1.3). The same choice of $\varepsilon$ also yields the right hand side. Finally we note that with our choice of $\varepsilon>0$ condition (3.7) amounts to (1.2).

As already mentioned, the "moreover part" follows from the fact that in this case $\alpha$ is an absolute constant. This completes the proof.

As an example, let us consider the case when $E=\ell_{2}^{k}$ for an appropriate dimension $k$. Since $E$ has cotype 2 with constant 1 our assumptions hold, and we obtain that for any isotropic, subgaussian vector $X$, the random operator $\Gamma=\sum_{i=1}^{k}\left\langle X_{i}, \cdot\right\rangle e_{i}$ satisfies

$$
c_{1} \sqrt{k} \leq\|\Gamma t\| \leq c_{2} \sqrt{k}
$$


whenever $\ell_{*}(T) \leq c_{3} \sqrt{k} / \log k$ (and in the Gaussian or Rademacher cases, the logarithmic term can be removed). This can be viewed as a weaker, "isomorphic" version of the main result in $[\mathrm{KM}]$ (see also [MPT]). Of course, the proof in $[\mathrm{KM}]$ uses the fact that $E$ is Euclidean in a very strong way while the main idea here is that the proof should work for an arbitrary space with cotype.

Another noteworthy point that follows from the proof is that if $X$ is a Gaussian vector, one can obtain a version of Theorem 1.1. In this case, as was noted in Remark 3.4, the use of the majorizing measure theorem in Lemma 3.3 can be replaced by Chevet's inequality (or Slepian's lemma). However, this argument gives worse dependence on $\varepsilon>0$, namely, the main assumption (1.1) must be satisfied with $\varepsilon^{2}$ replacing $\varepsilon$.

\section{References}

[DG] V. H. de la Pena and E. Giné, Decoupling, Springer-Verlag, New York, 1999.

[F] X. Fernique, Régularité des trajectoires des fonctiones aléatoires gaussiennes, in Ecole d'Eté deProbabilités de St-Flour 1974, Lecture Notes in Mathematics 480, SpringerVerlag, 1975, pp. 1-96.

[G1] Y. Gordon, Some inequalities for Gaussian processes and applications, Israel Journal of Mathematics, 50 (1985), 265-289.

[G2] Y. Gordon, On Milman's inequality and random spaces which escape through a mesh, in Geometric Aspects of Funtional Analysis (1986/87), Lectures Notes in Mathematics, 1988, pp. 84-106.

[G3] Y. Gordon, A note on an observation of G. Schechtman, in Geometric Aspects of Funtional Analysis (1986/87) Lectures Notes in Mathematics, in print.

[JM] N. C. Jain and M. B. Marcus, Continuity of sub-Gaussian processes, in Probability on Banach spaces, Adv. Probab. Related Topics, 4, 1978, pp. 81-196.

$[\mathrm{KM}]$ B. Klartag, and S. Mendelson, Empirical processes and random projections, Journal of Functional Analysis, 225 (2005), 229-245.

[L] M. Ledoux, The concentration of measure phenomenon, Mathematical Surveys an Monographs Vol 89, AMS, 2001.

[LTa] M. Ledoux and M. Talagrand, Probability in Banach spaces, Isoperimetry and processes, Ergebnisse der Mathematik und ihrer Grenzgebiete (3), vol. 23. SpringerVerlag, Berlin, 1991.

[MaPi] B. Maurey and G. Pisier, Séries de variables aléatoires vectorielles indépendantes et propriétés géométriques des espaces de Banach, Studia Mathematica, 58 (1976), 45-90.

[MPT] S. Mendelson, A. Pajor and N. Tomczak-Jaegermann, Reconstruction and subgaussian operators, in Asymptotic Geometric Analysis, Geometric and Functional Analysis, 17 (2007), 1248-1282. 
[MS] V. D. Milman and G. Schechtman, Asymptotic Theory of Finite Dimensional Normed Spaces, Lecture Notes in Mathematics, 1200, Springer 1986.

[Pi1] G. Pisier, Some applications of the metric entropy condition to harmonic analysis, in Lecture Notes in Mathematics, 995, Springer, Berlin, 1983, pp. 123-154.

[Pi2] G. Pisier, Probabilistic methods in the geometry of Banach spaces, in Probability and analysis (Varenna, 1985), Lecture Notes in Mathematics, 1206, Springer, Berlin, 1986, pp. $167-241$.

[Pi3] G. Pisier, The Volume of Convex Bodies and Banach Space Geometry, Cambridge University Press, Cambridge, 1989.

[Pi4] G. Pisier, Private communication.

[S] G. Schechtman, Two observations regarding embedding subsets of Euclidean spaces in normed spaces, Advances in Mathematics 200 (2006), 125-135.

[Ta1] M. Talagrand, Regularity of Gaussian processes, Acta Mathematica 159 (1987), 99-149.

[Ta2] M. Talagrand, Concentration of measure and isoperimetric inequalities in product spaces, Publications Mathématiques. Institut de Hautes Études Scientifiques, 81 (1995), 73-205.

[Ta3] M. Talagrand, M. The Generic Chaining. Upper and Lower Bound of Stochastic Processes, Springer Monographs in Mathematics, Springer-Verlag, Berlin, 2005.

[VW] A. W. Van der Vaart and J. A. Wellner, Weak Convergence and Empirical Processes, Springer Series in Statistics, Springer Verlag, New York, 1996. 